\title{
Late-Ensemble of Convolutional Neural Networks with Test Time Augmentation for Chest XR COVID-19 Detection
}

\author{
Abdul Qayyum, Imran Razzak, Moona Mazher, Domenec Puig \\ University of Burgundy, Dijon, France \\ Deakin University Geelong, Australia \\ University Rovira i Virgili, Spain \\ Email: \{engr.qayyum, mirpak, moona.mazher\}@gmail.com, domenec.puig@urv.cat
}

\begin{abstract}
COVID-19, a severe acute respiratory syndrome aggressively spread among global populations in just a few months. Since then, it has had four dominant variants (Alpha, Beta, Gamma and Delta) that are far more contagious than original. Accurate and timely diagnosis of COVID-19 is critical for analysis of damage to lungs, treatment, as well as quarantine management [7]. CT, MRI or X-rays image analysis using deep learning provide an efficient and accurate diagnosis of COVID-19 that could help to counter its outbreak. With the aim to provide efficient multi-class COVID-19 detection, recently, COVID-19 Detection challenge using $X$-ray is organized [12]. In this paper, the late-fusion of features is extracted from pre-trained various convolutional neural networks and fine-tuned these models using the challenge dataset. The DensNet201 with Adam optimizer and EffecientNet-B3 are fine-tuned on the challenge dataset and ensembles the features to get the final prediction. Besides, we also considered the test time augmentation technique after the late-ensembling approach to further improve the performance of our proposed solution. Evaluation on Chest XR COVID-19 showed that our model achieved overall accuracy is $95.67 \%$. We made the code is publicly available ${ }^{1}$. The proposed approach was ranked 6th in Chest XR COVID-19 detection Challenge [1].
\end{abstract}

Index Terms-COVID-19, Chest XR COVID-19, COVID-19 Challenge, Ensemble, X-Ray.

\section{INTRODUCTION}

COVID-19 is an infectious disease caused by the SARSCoV-2 virus that shares similarities with SARS and MERS viruses that were previously reported in 2003 and 2012. It is ongoing life threatening virus that the world has been facing since 2019. As of October 2021, COVID-19 has four dominant variants spreading among global populations: Alpha /B.1.1.7, first found in UK (London and Kent), Beta /B.1.351 found in South Africa, Gamma/P.1 found in Brazil Variant and the Delta/B.1.617.2 found in India Variant. Among these, Delta is one of the most contagious and deadly that has comparatively high high infectivity and extreme lethality. Up to 30 October 2021, 246 million COVID cases have been diagnosed, with $4.99 \mathrm{M}$ deaths worldwide. United States and India are the two leading countries that affected most with

\footnotetext{
${ }^{1}$ https://github.com/RespectKnowledge/Chest-XR-COVID-19detection $_{D}$ eep - Learning
}

45.9M/0.745M and 34.2/0.457M with cases/death. Figure $1^{2}$ shows the COVID-19 cases in France and Australia on 7th October 2021. Recently, deep learning has been widely applied in health especially in analyzing the images i.e. Chest images (MR, CIT and Ultrasound) for COVID image analysis [3], [4], [8], [13]. Qayuim et al. presented depth-wise neural network for COVID-19 diagnosis using CT image [10], [11]. They have uniformly scale all the dimensions and performed multilevel feature embedding result in increase feature representation. ConvNets upscaling is considered by balancing the scaling factor. Depth wise multilevel concatenation is applied to combine different features representation levels that improve diagnostic performance. In another work, Wang et al. proposed noise robust dice loss approach for lesion segmentation ensemble networks (COPLE-Net) for the segmentation of COVID19 [13]. Muhammad et al. presented a convolutionary neural network with much lower number of learning parameters. The network has five main layers of convolution connectors with multi-layer fusion functionality in each block, resulting in detection performance. Experiments on a publicly dataset showed $92.5 \%$ precision and $91.8 \%$ accuracy [6]. Wang et al. applied self-created $\mathrm{CNN}$ to learn individual image-level representations, and rank-based average pooling and multipleway data augmentation are applied to improve the diagnostic performance [14].

GNN Graph neural network is deployed to learn relationaware representations, and deep feature level fusion is performed to fuse individual image-level features and relationaware features from both $\mathrm{CNN}$ and GNN. Hussain et al. applied CNN named CoroDet for COVID-19 diagnosis using raw chest X-ray and CT. CoroDet showed efficient diagnostic performance in differentiating COVID, and Normal pneumonia patient [5]. CorDet showed a significant gain in performance and achieved $99.1 \%, 94.2 \%$ and $91.2 \$$ classification accuracy for for 2 class, 3, and 4 class classification. Abbas et al. presented DeTraC (Decompose, Transfer, and Compose) for the classification of COVID patient [2] as a results, the model is able to deal with any irregularities by first investigating

${ }^{2}$ https://www.worldometers.info/coronavirus/ 

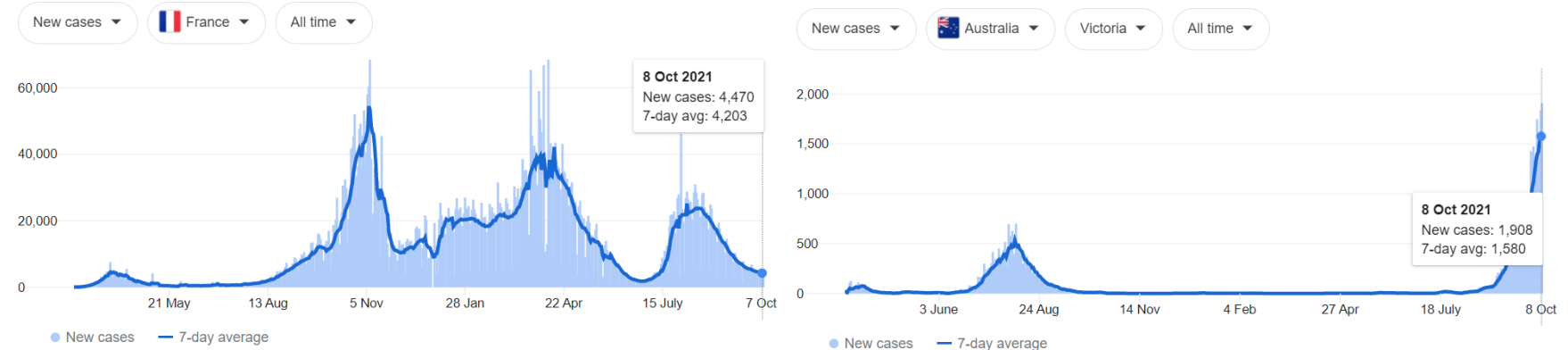

Fig. 1. Growth of COVID-19 Cases in France and Australia: Spikes in COVID cases shows COVID-19 1st, 2nd and 3rd wave

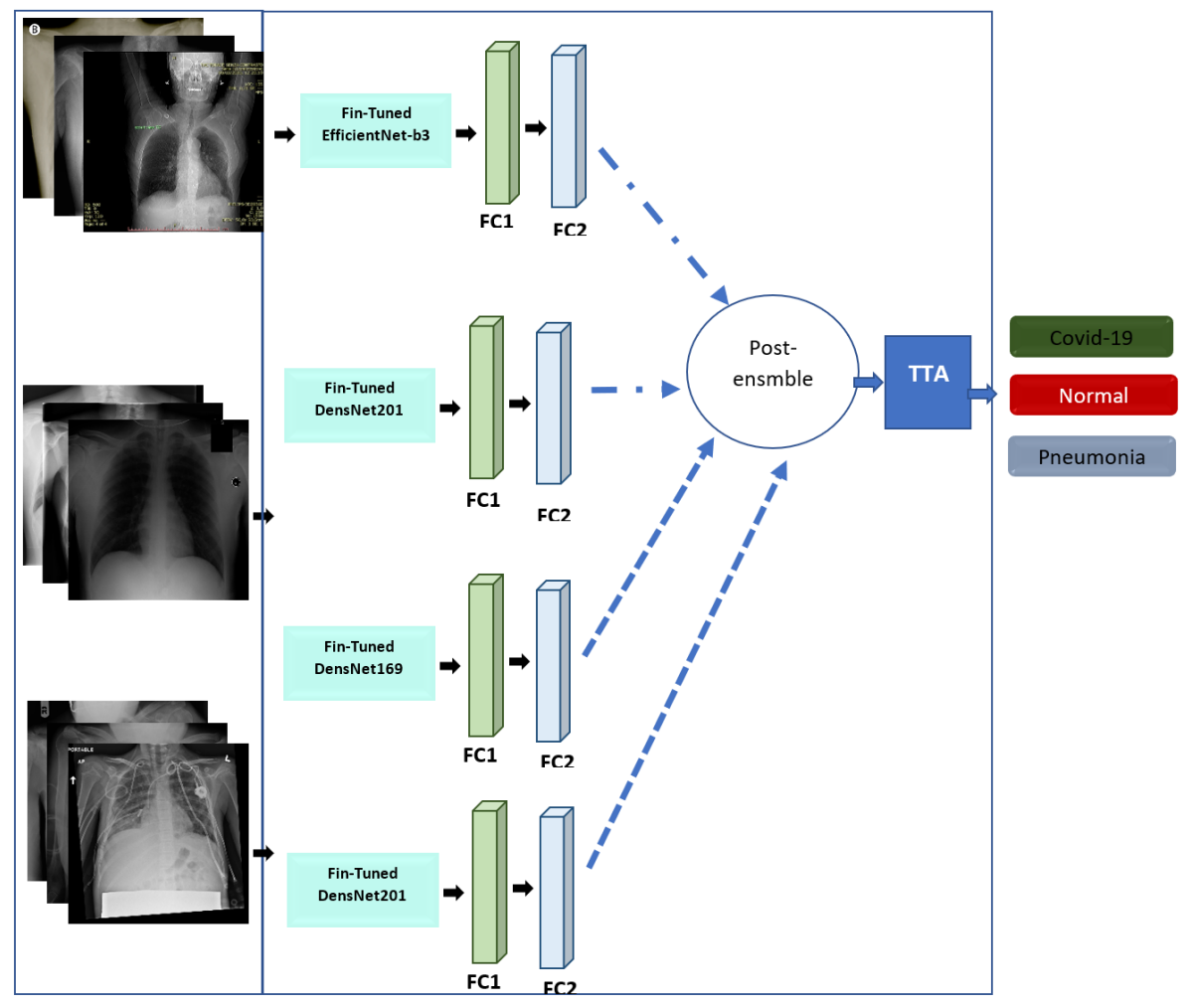

Fig. 2. Proposed Late-Ensemble Network

the boundary of the target class. Results showed that DeTraC achieved $93.1 \%$ and $100 \%$ sensitivity for COVID-19 diagnosis. In another work, Zebin et al. used multiple pre-trained models as feature extractors and achieved of 90\%, 94.3\% , and $96.8 \%$ over all accuracy using the VGG16, ResNet50, and EfficientNetB0, respectively [16]. Besides, the generative adversarial framework is also utilized to deal with minority COVID-19 class. Phasm fine-tuned alexNet, GoogleNet, and SqueezeNet without data augmentation for 2-class and 3-class COVID19 diagnosis [9]. Wang et al. presented a computer aided diagnostic framework that consists of Discrimination-deep learning and Localization-deep learning [15]. Discriminationdeep learning extracts lung features from chest X-ray images followed by training. Followed by Localization-deep learning which was trained with 406-pixel patches to extract the recognized of X-ray images and classify them to left lung, right lung or bipulmonary. X-ray. The two-layered approach showed better performance than radiologists and achieved $98.71 \%$ and $93.03 \%$ accuracy, classification and localization, respectively. To provide effective and efficient COVID-19 diagnosis, one of the vital effort is Chest XR COVID-19 Chest X-ray image classification Challenge. In this paper, we presented late ensemble convolutional neural networks with test time augmentation for detecting COVID-19 affected patient using chest X-ray images. Experiments were conduction on the Chest XR COVID-19 detection challenge dataset. We have used proposed models trained weights to average the features in post ensemble using mean average voting technique 


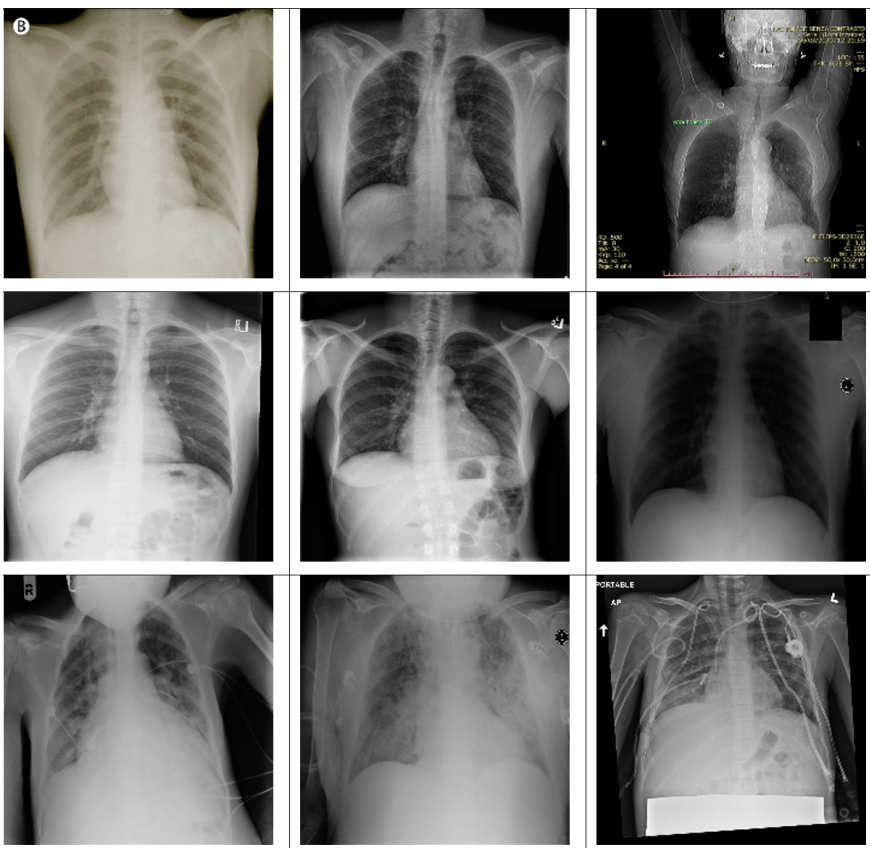

Fig. 3. First row-COVID-19 samples, second row-Normal and third rowPneumonia

and then passed to the Test Time augmentation technique. Evaluation of the test dataset showed that our model had achieved significantly better performance.

\section{Methodology: Ensemble Network}

In this section, we present the proposed deep learning framework for COVID-19 Diagnosis. Figure 2 illustrate the proposed ensemble framework. To improve the diagnostic performance, we have trained various pretrained models such as DensNet121, DensNet161, DensNet201, ResNet18,ResNet34, EfficienNet-b0-B7, ResNext50, ResNext101 and MobileNetV2. We have fine-tuned the weights of each model using the Challenge dataset based on a pre-trained convolutional neural network. We have selected best-performing methods and performed late ensembling. To improve the performance, we applied different data augmentation (horizontalFlip and verticalFlip). Finally, we have used models trained weights to average the features in post ensemble using mean average voting technique and then passed to the Test Time augmentation technique. Similar to what data augmentation is doing to the training set, the purpose of test time augmentation is to perform random modifications to the test images. Thus, instead of showing the regular, "clean" images only once to the trained model, we show the augmented images several times and then average the predictions of each corresponding image and take that as the final prediction. We used horizontal and vertical flipping as test time augmentation in our proposed solution and received fruitful results.

\section{RESUlts AND Discussion}

In this section, we provide the dataset, parameter and experimental setup, and results. In order to compare the performance, we have used accuracy, sensitivity, and specificity.

\section{A. Dataset}

Int this experiment, we have used Chest XR COVID-19 detection challenge dataset [1]. Chest XR COVID-19 is a large-scale multiclass (COVID-19, Normal and Pneumonia) Chest X-ray image classification dataset. Figure 3 shows COVID-19, Pneumonia and healthy images from dataset. The dataset consist of 20,000+ images which are divided into train (17,955 images) and validation (3,430 images) sets.

\section{B. Parameters}

In this experiment, we have used PyTorch library for model development. The proposed model was trained with different hyperparameters. We set the learning rate to 0.0001 with Adam,adamx, adamW optimizers. As the dataset is imbalanced classes samples, the weighted cross-entropy function is used as a loss function between the output of the model and the ground-truth sample. The inverse class frequencies for weight balancing have been used to calculate the weighted crossentropy loss function. The higher-class samples require less weight and fewer class samples need more weight values. The 32 batch-size with 500 epochs has been used with 15 early stopping steps. The best model weights have been saved for prediction in the validation phase. The $224 \times 224$ input image size was used for training and prediction. We have used the V100 tesla NVidia-GPU machine for training and testing the proposed model. The detail of the training protocol is described in I.

\section{Discussion}

In this paper, we have fine-tune and performed late ensemble of state of the art performing networks such as DensNet121, DensNet161, DensNet201, ResNet18,ResNet34, EfficienNetb0-B7, ResNext50, ResNext101 and MobileNetV2. Experiments were conducted on the Challenge XR dataset. Table III shows the performance of different methods. Notice that we have ensembled different combinations of networks (independent efficientNet, DenseNet, and ensemble of two, three, and four networks. To improve the performance, we applied different data augmentation (horizontalFlip and verticalFlip). Figure 4 shows the feature extracted from last fully connected layers for three classes. We used TSNE to plot high dimensions features into 2-dimensional. We can observe that ensembles of four networks (Figure (a)) showed that very few features overlap comparatively with other class features.

Experimental results showed that the ensemble of four networks showed 95.67\% , 95.67\%, and 95.77\% accuracy, sensitivity and specificity, respectively. We can observe that the combination of three models shows slightly lower performance than the ensemble of three network $(95.58 \%, 95.58 \%$, and $95.63 \%$ respectively ). Similar trend can be noticed with single network. We can observe that EfficientNet and DenseNet showed similar performance. The individual DensNet201 with admax optimzer produced 0.9475 accuracy as compared to single DensNet201 with adam optimizer. To improve the 
medRxiv preprint doi: https://doi.org/10.1101/2022.02.25.22271520; this version posted February 26, 2022. The copyright holder for this preprint (which was not certified by peer review) is the author/funder, who has granted medRxiv a license to display the preprint in perpetuity.

\section{It is made available under a CC-BY-NC-ND 4.0 International license .}

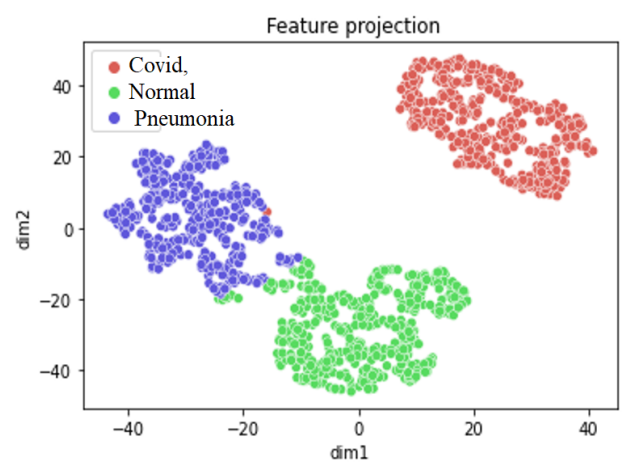

(a) Proposed models(4 ensembled)

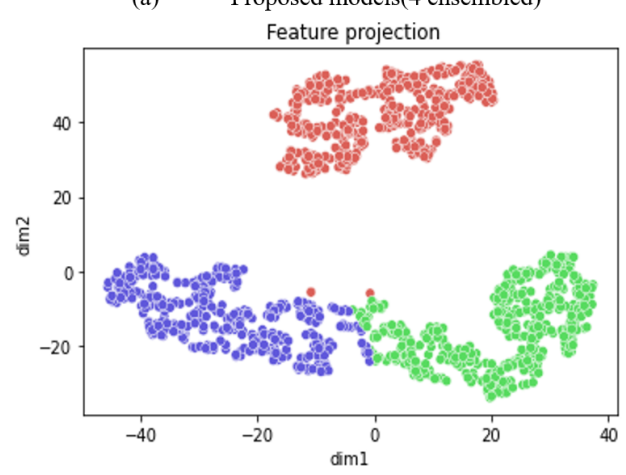

(c) Proposed models (2ensembled)

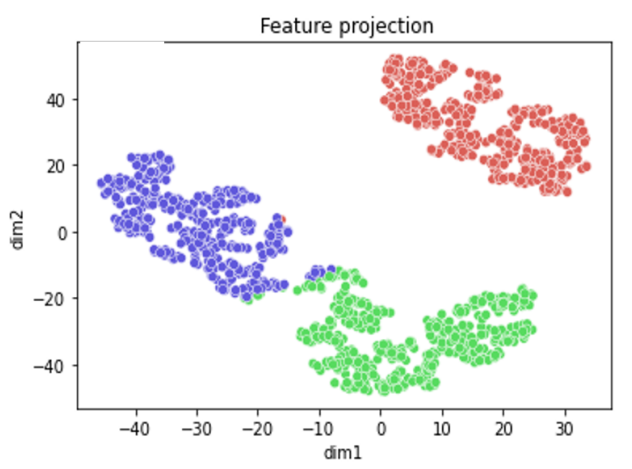

(b) Proposed models(3 ensembled)

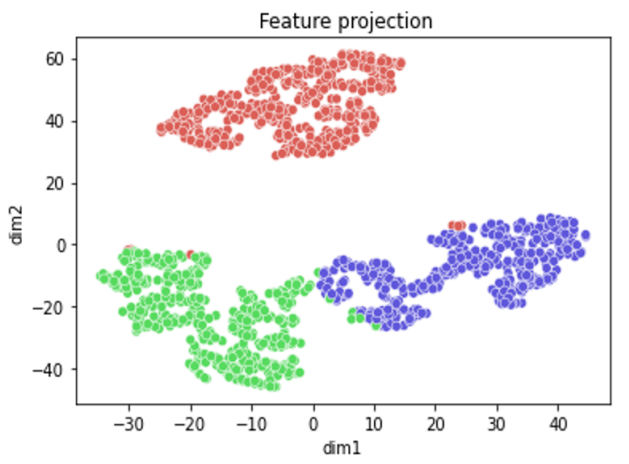

(d) Proposed models (single model)

Fig. 4. Feature projection of logits layer on test dataset for three classes using proposed models. Class 0 represented covid, 1 represnted normal and 2 represented Pneumonia

TABLE I

NETWORK PARAMETERS

\begin{tabular}{|l|l|}
\hline \hline Data augmentation methods & HorizontalFlip $(\mathrm{p}=0.5)$, VerticalFlip $(\mathrm{p}=0.5)$, RandomBrightnessContrast (p=0.8) \\
\hline Initialization of the network & "he" normal initialization \\
\hline Batch size & 32 \\
\hline Patch size & $224 \times 224$ \\
\hline Total epochs & 500 \\
\hline Optimizer & Adam, Adamx, AdamW \\
\hline Initial learning rate & 0.0001 \\
\hline Learning rate decay schedule & None \\
\hline Stopping criteria, and optimal model selection criteria & The stopping criterion is reaching the maximum number of epoch \\
\hline \hline
\end{tabular}

TABLE II

COMPARATIVE EVALUATION OF DiFFERENT NeTWORK ON VALIDATION SET

\begin{tabular}{|l|l|l|l|}
\hline Models & Accuracy & Sensitivity & Specificity \\
\hline Ensemble-four models with TTA & 0.9687 & 0.968 & 0.9691 \\
\hline Ensemble-three models with TTA & 0.9662 & 0.9613 & 0.9687 \\
\hline Ensemble-two models with TTA & 0.9644 & 0.9617 & 0.9633 \\
\hline Ensemble-two without TTA & 0.9613 & 0.9610 & 0.9641 \\
\hline EfficieNet-b3 & 0.9562 & 0.9560 & 0.9565 \\
\hline DensNet201(adam optimizer) & 0.9567 & 0.9558 & 0.9542 \\
\hline RensNet201(adamax optimizer) & 0.9513 & 0.9527 & 0.9545 \\
\hline DensNet201 & 0.9412 & 0.9413 & 0.9421 \\
\hline
\end{tabular}

performance, we applied different data test time augmentation, which helped to improve the performance slightly. The proposed solution showed high accuracy on the test dataset; however, it is computational complex in prediction due to test time augmentation. In the future, we will try to reduce the test or inference time by either reducing the parameters of the proposed model.

\section{CONCLUSION}

The exponential growth in COVID cases is overwhelming health-care system. Every patient with respiratory illness cannot be tested using conventional techniques with limited testing kits. This paper presented late ensemble convolutional neural networks with test time augmentation for detecting COVID-19 affected patients using chest X-ray images. Experiments were conduction on the Chest XR COVID-19 detection challenge dataset. Experimental results on a large-scale dataset showed that our late ensemble networks achieved $95.67 \%$ 
TABLE III

Comparative Evaluation of Different Network on Test Set

\begin{tabular}{|l|l|l|l|}
\hline Models & Accuracy & Sensitivity & Specificity \\
\hline Ensemble-four models with TTA & $\mathbf{0 . 9 5 6 7}$ & $\mathbf{0 . 9 5 6 7}$ & $\mathbf{0 . 9 5 7 7}$ \\
\hline Ensemble-three models with TTA & 0.9558 & 0.9558 & 0.9563 \\
\hline Ensemble-two models with TTA & 0.9558 & 0.9558 & 0.9559 \\
\hline Ensemble-two without TTA & 0.9525 & 0.9525 & 0.9539 \\
\hline EfficieNet-b3 & 0.9450 & 0.9450 & 0.9455 \\
\hline DensNet201(adam optimizer) & 0.9458 & 0.9458 & 0.9462 \\
\hline RensNet201(adamax optimizer) & 0.9475 & 0.9475 & 0.9484 \\
\hline DensNet201 & 0.9300 & 0.9300 & 0.9308 \\
\hline
\end{tabular}

, 95.67\%, and 95.77\% accuracy, sensitivity, and specificity, respectively. The proposed approach was ranked 6th in Chest XR COVID-19 detection Challenge [1]

\section{REFERENCES}

[1] Moulay A. Akhloufi and Mohamed Chetoui. Chest XR COVID19 detection. https://cxr-covid19.grand-challenge.org/, August 2021. Online; accessed September 2021.

[2] Sohaib Asif, Yi Wenhui, Hou Jin, and Si Jinhai. Classification of covid-19 from chest $\mathrm{x}$-ray images using deep convolutional neural network. In 2020 IEEE 6th International Conference on Computer and Communications (ICCC), pages 426-433. IEEE, 2020.

[3] Jannis Born, Nina Wiedemann, Gabriel Brändle, Charlotte Buhre, Bastian Rieck, and Karsten Borgwardt. Accelerating covid-19 differential diagnosis with explainable ultrasound image analysis. arXiv preprint arXiv:2009.06116, 2020.

[4] Deng-Ping Fan, Tao Zhou, Ge-Peng Ji, Yi Zhou, Geng Chen, Huazhu Fu, Jianbing Shen, and Ling Shao. Inf-net: Automatic covid-19 lung infection segmentation from ct images. IEEE Transactions on Medical Imaging, 2020.

[5] Emtiaz Hussain, Mahmudul Hasan, Md Anisur Rahman, Ickjai Lee, Tasmi Tamanna, and Mohammad Zavid Parvez. Corodet: A deep learning based classification for covid-19 detection using chest x-ray images. Chaos, Solitons Fractals, 142:110495, 2021.

[6] Ghulam Muhammad and M. Shamim Hossain. Covid-19 and non-covid19 classification using multi-layers fusion from lung ultrasound images. Information Fusion, 72:80-88, 2021.

[7] Usman Naseem, Imran Razzak, Matloob Khushi, Peter W Eklund, and Jinman Kim. Covidsenti: A large-scale benchmark twitter data set for covid-19 sentiment analysis. IEEE Transactions on Computational Social Systems, 8(4):1003-1015, 2021.

[8] Nidal Nasser, Qazi Emad-ul Haq, Muhammad Imran, Asmaa Ali, Imran Razzak, and Abdulaziz Al-Helali. A smart healthcare framework for detection and monitoring of covid-19 using iot and cloud computing. Neural Computing and Applications, pages 1-15, 2021.

[9] Tuan D Pham. Classification of covid-19 chest x-rays with deep learning: new models or fine tuning? Health Information Science and Systems, 9(1):1-11, 2021.

[10] Abdul Qayyum, Mona Mazhar, Imran Razzak, and Mohamed Reda Bouadjenek. Multilevel depth-wise context attention network with atrous mechanism for segmentation of covid19 affected regions. Neural Computing and Applications, pages 1-13, 2021.

[11] Abdul Qayyum, Imran Razzak, M Tanveer, and Ajay Kumar. Depthwise dense neural network for automatic covid19 infection detection and diagnosis. Annals of operations research, pages 1-21, 2021.

[12] Arshia Rehman, Saeeda Naz, Ahmed Khan, Ahmad Zaib, and Imran Razzak. Improving coronavirus (covid-19) diagnosis using deep transfer learning. MedRxiv, 2020.

[13] Guotai Wang, Xinglong Liu, Chaoping Li, Zhiyong Xu, Jiugen Ruan, Haifeng Zhu, Tao Meng, Kang Li, Ning Huang, and Shaoting Zhang. A noise-robust framework for automatic segmentation of covid-19 pneumonia lesions from ct images. IEEE Transactions on Medical Imaging, 39(8):2653-2663, 2020.

[14] Shui-Hua Wang, Deepak Ranjan Nayak, David S. Guttery, Xin Zhang, and Yu-Dong Zhang. Covid-19 classification by ccshnet with deep fusion using transfer learning and discriminant correlation analysis. Information Fusion, 68:131-148, 2021.
[15] Zheng Wang, Ying Xiao, Yong Li, Jie Zhang, Fanggen Lu, Muzhou Hou, and Xiaowei Liu. Automatically discriminating and localizing covid-19 from community-acquired pneumonia on chest x-rays. Pattern Recognition, 110:107613, 2021.

[16] Tahmina Zebin and Shahadate Rezvy. Covid-19 detection and disease progression visualization: Deep learning on chest x-rays for classification and coarse localization. Applied Intelligence, 51(2):1010-1021, 2021. 